\title{
Acknowledgement to Reviewers of Journal of Low Power Electronics and Applications in 2016
}

\author{
Journal of Low Power Electronics and Applications Editorial Office \\ Published: 10 January 2017 \\ MDPI AG, St. Alban-Anlage 66, 4052 Basel, Switzerland; jlpea@mdpi.com
}

The editors of Journal of Low Power Electronics and Applications would like to express their sincere gratitude to the following reviewers for assessing manuscripts in 2016.

We greatly appreciate the contribution of expert reviewers, which is crucial to the journal's editorial process. We aim to recognize reviewer contributions through several mechanisms, of which the annual publication of reviewer names is one. Reviewers receive a voucher entitling them to a discount on their next MDPI publication and can download a certificate of recognition directly from our submission system. Additionally, reviewers can sign up to the service Publons (https://publons.com) to receive recognition. Of course, in these initiatives we are careful not to compromise reviewer confidentiality. Many reviewers see their work as a voluntary and often unseen part of their role as researchers. We are grateful to the time reviewers donate to our journals and the contribution they make.

If you are interested in becoming a reviewer for JLPEA, see the link at the bottom of the webpage http://www.mdpi.com/reviewers.

The following reviewed for JLPEA in 2016:

\author{
Abbas, Zia \\ Akopyan, Filipp A. \\ Anders, Jens \\ Bailey, Donald \\ Banik, Subhadeep \\ Becker, Georg T. \\ Bong, David Boon Liang \\ Bosio, Alberto \\ Bystrov, Alex \\ Chan, Pak Kwong \\ Chang, Meng-Fan (Marvin) \\ Chung, Ching-Che \\ Claesen, Luc \\ Crupi, Felice \\ Czornomaz, Lukas \\ Dabrowski, Jerzy \\ El-Damak, Dina \\ Fallahzadeh, Ramin \\ Flandre, Denis \\ Graham, David W. \\ Heuser, Annelie \\ Horng, Jiun-Wei \\ Huang, Shu-Chuan \\ Jurišić, Dražen \\ Kaminaga, Masahiro \\ Kang, Seung H.
}

Karlin, Ian
kavehei, Omid
Kim, SangHyeon
Kitsos, Paris
Koeune, François
Li, Na
Liao, Huailin
Liao, Ming Han
Liu, Yongpan
Lorenz, Michael G.
Lu, Chao
Lu, Yan
Lukowiak, Marcin
Maruta, Kazuki
Michel, Fridolin
Mitsugi, Jin
Moll, Francesc
Mols, Yves
Moradi, Saber
Oswald, David
Oswald, Elisabeth
Paidimarri, Arun
Palesi, Maurizio
Papaioannou, Thanasis
Park, Jongsun
Park, Sangyoung

Pehl, Michael

Peng, Sheng-Yu

Qiao, Ning

Richelli, Anna

Roger, Sandra

Shafik, Rishad

Shrivastava, Aatmesh

Song, Minkyu

Sun, Guangyu

Swabey, Matthew

Teh, Ying-Khai

Tehranipoor, Mark M.

Van Breussegem, Tom

Van Helleputte, Nick

Vătăjelu, Elena Ioana

Wang, Peng-Fei

Weizman, Yoav

Wong, Ming Ming

Yang, Hao-I

Yokoyama, Yoshisato

Zhan, Chenchang

Zhang, Rui

Zhang, Wei

Zhao, Weisheng 
(C) 2017 by the authors; licensee MDPI, Basel, Switzerland. This article is an open access article distributed under the terms and conditions of the Creative Commons Attribution (CC-BY) license (http://creativecommons.org/licenses/by/4.0/). 